\title{
Investigation of the Hammerstein Hypothesis in the Modeling of Electrically Stimulated Muscle
}

\author{
Kenneth J. Hunt,* Member, IEEE, Marko Munih, Member, IEEE, Nick de N. Donaldson, and Fiona M. D. Barr
}

\begin{abstract}
To restore functional use of paralyzed muscles by automatically controlled stimulation, an accurate quantitative model of the stimulated muscles is desirable. The most commonly used model for isometric muscle has had a Hammerstein structure, in which a linear dynamic block is preceded by a static nonlinear function. To investigate the accuracy of the Hammerstein model, the responses to a pseudo-random binary sequence (PRBS) excitation of normal human plantarflexors, stimulated with surface electrodes, were used to identify a Hammerstein model but also four local models which describe the responses to small signals at different mean levels of activation. Comparison of the local models with the linearized Hammerstein model showed that the Hammerstein model concealed a fivefold variation in the speed of response. Also, the small-signal gain of the Hammerstein model was in error by factors up to three. We conclude that, despite the past widespread use of the Hammerstein model, it is not an accurate representation of isometric muscle. On the other hand, local models, which are more accurate predictors, can be identified from the responses to short PRBS sequences. The utility of local models for controller design is discussed.
\end{abstract}

Index Terms-Electrostimulation, model identification, muscle, rehabilitation.

\section{INTRODUCTION}

$\mathbf{T}$ HE FORCE produced by an electrically stimulated muscle depends on the stimulus intensity and the stimulus frequency. Intensity determines, in a nonlinear fashion, which neurons are depolarized by the stimulus pulse and, therefore, which motor units in the muscles contract. In models of stimulated isometric muscles, it is common to represent the nerve-muscle as a two-block structure: the first block being a time-independent nonlinear function representing the recruitment, and the second block being the muscle contraction dynamics. This structure has been used so often that it has become orthodox.

However, one might expect it to be a poor model for the following reason. According to Henneman's principle, for normal neuromuscular systems, smaller motor nerve fibers innervate slow motor units and vice versa [1]. It is also the case that with short rectangular stimulation pulse currents, nerve

Manuscript received July 15, 1996; revised February 23, 1998. Asterisk indicates corresponding author.

*K. J. Hunt is with the Centre for Systems and Control, Department of Mechanical Engineering, University of Glasgow, Glasgow G12 8QQ Scotland (e-mail: k.hunt@mech.gla.ac.uk).

M. Munih is with the Faculty of Electrical Engineering, University of Ljubljana, Ljubljana, Slovenia.

N. de Donaldson is with the Department of Medical Physics and Bioengineering, University College London, London WCIE 6AJ England.

F. M. D. Barr is with the Royal National Orthopaedic Hospital Trust, Stanmore, England.

Publisher Item Identifier S 0018-9294(98)05322-1. fibers are recruited in the reverse order of their diameters ("nonphysiological recruitment") [2]. We, therefore, expect that dynamics will become slower as the intensity increases. This cannot be represented by the two-block model which assumes that the dynamics are independent of intensity.

Nonlinear models of electrically stimulated muscle have been reviewed by Durfee [3]. Block models are only one of several possible structures which can be used in biological (i.e., nonlinear) system identification [4]. Where there are only two blocks, one of which is nonlinear, the nonlinearity may be placed before or after the dynamic block, in, respectively, so-called Hammerstein [5] or Wiener forms. While techniques for indentifying the parameters for these models have been investigated by Hunter and Kearney [6], those working in the field of functional electrical stimulation have usually adopted the Hammerstein structure [7]-[11]. No doubt this is due to its correspondence to the biophysics: the two blocks representing the nerve fiber recruitment followed by muscle contraction dynamics. Ease of parameter identification is also important [12]. The need to neutralize the nonlinearity is obvious in the crudest measurements of muscle response where a deadband and saturation are evident. However, despite this popularity, the intensity-dependence of the muscle dynamics has long been known [13], so clearly it is desirable to assess the deficiencies of the Hammerstein model and to consider how the model could be improved without making parameter identification impractical.

In this paper, we begin by identifying a Hammerstein model of the plantarflexors of an intact human, stimulated by surface electrodes. We used the twitch response test of Durfee and Maclean [12] to determine the recruitment curve, the nonlinear block. Thereafter, a pseudo-random binary sequence (PRBS) activation signal was used to stimulate the muscle. The best order and time delay for the discrete-time linear transfer function were judged by comparing the response of the transfer function as a predictor to experimental responses which had not been used for identification. It appeared that the PRBS signal gives a model which is a better predictor than the twitch responses. We then used small-amplitude PRBS excitation, at four different mean levels of activation, to obtain local models. These local models are significantly different from each other, and from the Hammerstein model, showing, as expected, that the latter is not an accurate representation. This suggests that stimulation controllers based on local models may give significantly better results.

All experiments described in this paper are part of a series in which we are investigating the behavior of the ankle joints as actuators for artificial motor control. With the "wobbler" 
apparatus, we can measure joint stiffness or identify the isometric moment responses of the feet to stimulation of the plantarflexors [14]. We can then use this data to design controllers to "stand" the subject using ankle control while the other joints are braced [15]-[17]. Our preferred controllers use feedback of both the joint moment and the angle.

\section{EXPERIMENTAL PROCEDURES}

In this section, we briefly describe the experimental conditions, the wobbler apparatus, the muscles used, the measurement hardware and the software. The experimental estimation, and validation data sets and twitch responses were measured by electrically stimulating human plantarflexor muscles of a neurologically and physically intact man aged 44 .

Because we were identifying only ankle plantarflexor muscles, all his joints and segments above the ankles were fixed by strapping him into a special brace. The body was then a single inverted pendulum, which was held upright by light ropes from the left and right shoulders to a frame mounted below the ceiling. During the experiment, he placed both arms across his chest and did not interfere with stimulation voluntarily. The feet were placed in the Wobbler apparatus [14]. There are two foot boxes in this apparatus into which sports shoes are fixed. The boxes are mounted on a shaft with its rotational axis aligned with the joint centre for plantarflexion of the subject's ankles. The ankle moments were measured in isometric conditions by locking the shaft with the feet in a horizontal position. The moment resolution was $0.1 \mathrm{Nm}$. The left and right ankle moment data acquisition, stimulator command, and system control was performed on a PC computer with custom software.

The self-adhesive 50-mm-diameter Axelgaard electrodes ${ }^{1}$ were placed on the midlines of the soleus and gastrocnemius muscles of the subject. A multichannel Stanmore Stimulator [18] delivered monophasic charge-balanced constant-current pulses to one leg at a time. Pulse amplitude was preadjusted in setup Test A, increasing the current in 10-mA steps until a strong contraction resulted at $500-\mu$ s pulse width without significant pain. In these tests, $60 \mathrm{~mA}$ was used. Muscles were stimulated tetanically at $20 \mathrm{~Hz}$ with variable pulse-width in 2$\mu \mathrm{s}$ steps in the range from 0 to $500 \mu \mathrm{s}$. This pulse-width range represents a normalized muscle activation in the range from 0 to $1000 \mathrm{mAct}$ as is described further in the paper. The tests described were all done on one day in one leg (the left side).

Stimulation frequencies at or close to $20 \mathrm{~Hz}$ are often used because this rate is high enough for the contraction to be acceptably smooth. The stimulation rate cannot, however, be much higher than this as then the high rate of muscle fatigue would be exacerbated. This sampling frequency is also appropriate for typical bandwidth requirements in closed-loop control of muscle moment [15]-[17].

Two tests were used: in Test $B, 50$ twitch responses were elicited at 1.2-s intervals. These were five groups of ten pulses, in the range $[50,100, \cdots, 500] \mu$ s, which were applied in random order. The PC sampled at $200 \mathrm{~Hz}$ the left and right joint moments from torque load cells in the shaft and checked

\footnotetext{
${ }^{1}$ Axelgaard Manufacturing Company, Fallbrook, CA.
}

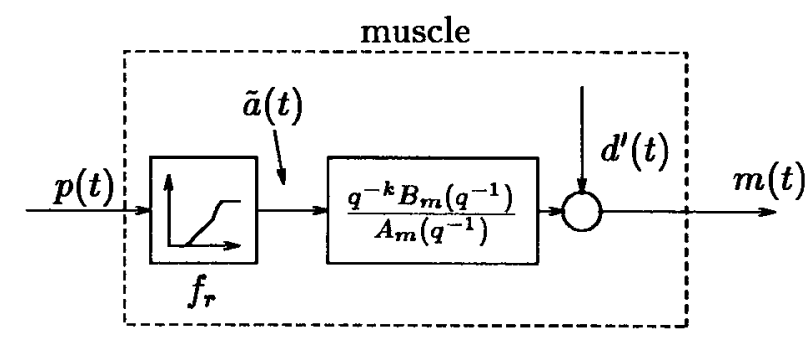

Fig. 1. Hammerstein model of muscle, $d^{\prime}(t)=d(t) / A_{m}\left(q^{-1}\right)$.

the exact time of pulse delivery. Later, the five moment responses at every pulse-width were averaged and the peak moment value was stored as one point of the Hammerstein recruitment curve. Data are further processed as described in Section IV.

Test PRBS was used to measure PRBS signal responses. A PRBS input sequence was generated with Matlab software in advance off-line, and stored in a file. PRBS signals are specified in mAct units (0-1000). The Test PRBS program read those signals from the file, converted to units of pulse width with the inverse recruitment curve, previously measured in Test B, and sent these in real time to the Stanmore Stimulator. (But see Section V, where the inverse recruitment curve was taken as linear.) Stimulation and sampling of the moments occurred at a constant $20-\mathrm{Hz}$ frequency. The delay in the system between an input value being read from the file and the stimulation pulse being delivered to the electrodes was 6.7 $\mathrm{ms}$, much less than the 50-ms interpulse interval. This delay was caused by the PC, serial communication to the stimulator and the stimulator's program.

\section{ESTIMATION AND VALIDATION AlgORITHMS}

\section{A. Procedure}

The open-loop muscle model in Hammerstein form is depicted in Fig. 1.

The signals in this model are as follows:

$m(t)$ muscle moment;

$\tilde{a}(t) \quad$ muscle "activation level";

$p(t) \quad$ stimulation pulse width (constant amplitude);

$d^{\prime}(t)$ disturbance signal.

In Hammerstein form the muscle consists of the static recruitment nonlinearity $f_{r}$ followed by the linear discrete-time transfer function $q^{-k} B_{m}\left(q^{-1}\right) / A_{m}\left(q^{-1}\right)$. The linear part of the model is assumed to be of auto-regressive with exogenous input (ARX)-type, i.e., it is described by

$$
A_{m}\left(q^{-1}\right) m(t)=q^{-k} B_{m}\left(q^{-1}\right) \tilde{a}(t)+d(t)
$$

where the net effect of disturbances is represented by the zeromean white noise signal $d$. (Note that (1) implies that the signal $d^{\prime}$ in Fig. 1 is related to $d$ by $d^{\prime}(t)=d(t) / A_{m}\left(q^{-1}\right)$.)

The integer $k \geq 1$ is a discrete input-output time-delay. $A_{m}$ and $B_{m}$ are polynomials in the delay operator $q^{-1}$ and have the form

$$
\begin{aligned}
& A_{m}\left(q^{-1}\right)=1+\alpha_{1} q^{-1}+\cdots+\alpha_{n a} q^{-n a} \\
& B_{m}\left(q^{-1}\right)=b_{0}+b_{1} q^{-1}+\cdots+b_{n b} q^{-n b}
\end{aligned}
$$




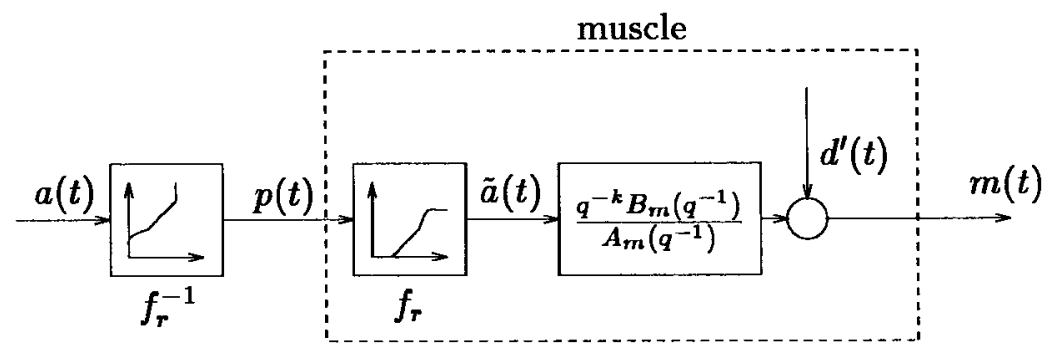

Fig. 2. Structure for global transfer-function identification in Hammerstein model, $d^{\prime}(t)=d(t) / A_{m}\left(q^{-1}\right)$.

Empirical determination of the Hammerstein model consists of two steps.

1) The impulse response method [12] is used to determine the recruitment nonlinearity $f_{r}$ based on twitch response data (the responses to well-separated stimulus pulses from which the muscle contraction or twitch is completed before the next stimulus pulse).

2) Parametric identification methods are used to determine the parameters of the linear transfer function. In this paper, a PRBS-stimulation test is employed to generate identification data.

The impulse response method is described in detail in Section IV-A. In order to generate test data for estimation of the linear part of the muscle model, the approximated recruitment nonlinearity $f_{r}$ from one. is first cancelled via the inverse function $f_{r}^{-1}$; the setup for identification of the linear transfer function is shown in Fig. 2.

A series of activation test signals $a(t)$ are applied (typically PRBS signals) and the ankle moment $m(t)$ is recorded.

\section{B. Parameter Estimation}

Assuming perfect cancellation of the recruitment nonlinearity the open-loop muscle can be represented by the model

$$
m(t)=\frac{q^{-k} B_{m}\left(q^{-1}\right)}{A_{m}\left(q^{-1}\right)} a(t)+\frac{1}{A_{m}\left(q^{-1}\right)} d(t)
$$

Since the disturbance $d$ is assumed to be an uncorrelated zero-mean stochastic signal, the best 1-step-ahead moment prediction is [19]

$$
\hat{m}(t)=\theta^{T} \phi(t-1)
$$

where the parameter vector $\theta$ and regression vector $\phi$ are defined by

$$
\begin{aligned}
\theta= & \left(\alpha_{1} \cdots \alpha_{n a} ; b_{0} \cdots b_{n b}\right)^{T} \\
\phi(t-1)= & (-m(t-1) \cdots-m(t-n a) \\
& a(t-k) \cdots a(t-k-n b))^{T} .
\end{aligned}
$$

A measure of model fidelity is provided by the least-squares criterion

$$
J_{N}(\theta)=\frac{1}{N} \sum_{t=1}^{N}(m(t)-\hat{m}(t))^{2}
$$

where $N$ is the number of data points. The analytical solution for the optimal parameter estimate is [19], [20]

$$
\hat{\theta}=\left(\sum_{t=1}^{N} \phi(t-1) \phi^{T}(t-1)\right)^{-1} \cdot\left(\sum_{t=1}^{N} \phi(t-1) m(t)\right)
$$

providing that the inverse exists. This is the solution used for parameter estimation in the identification experiments reported in the sequel.

\section{Model Validation}

For the validation of identified models, it is useful to use more general measures than the 1-step-ahead criterion (8), which tends to emphasize high-frequency components in the model. In this work, models are validated using a separate set of validation data with the $k^{p}$-step-ahead root-mean-square (rms) criterion

$$
J_{N}\left(k^{p}, \theta\right)=\left(\frac{1}{N} \sum_{t=1}^{N}\left(m(t)-\hat{m}\left(t \mid t-k^{p}\right)\right)^{2}\right)^{\frac{1}{2}} .
$$

Here, the $\mid$ operator denotes that the prediction $\hat{m}$ is conditional upon measured outputs available up to time $t-k^{p}$; the $k^{p}$ step-ahead moment prediction $\hat{m}\left(t \mid t-k^{p}\right)$ is defined by

$$
\hat{m}\left(t \mid t-k^{p}\right)=\theta^{T} \phi\left(t-1 \mid t-k^{p}\right)
$$

with the conditional regressor vector recursively calculated using

$$
\begin{aligned}
& \phi\left(t-1 \mid t-k^{p}\right) \\
& =\left(-\hat{m}\left(t-1 \mid t-k^{p}\right) \cdots-\hat{m}\left(t-k^{p}+1 \mid t-k^{p}\right)\right. \\
& \quad-m\left(t-k^{p}\right) \cdots-m(t-n a) \\
& \quad a(t-k) \cdots a(t-k-n b))^{T}
\end{aligned}
$$

Various values of $k^{p}$ are used in the validation tests.

An alternative method of measuring the frequency response is by Fourier transformation of the twitch responses [12]. In our experiments, however, we do not use Fourier methods to determine the nonparametric frequency responses. Instead, we use the parameter identification method described above, with PRBS-stimulation, since the parametric transfer-function models are required for the analytical control system design methods we employ for muscle moment control (see Hunt $e t$ al. [15], [21], and [22]). (For comparison, we also apply parametric identification to the twitch responses in Section IV-C.) 


\section{Imperfect Cancellation}

Note that in practice we can expect only imperfect cancellation of the recruitment nonlinearity. At a given operating point, imperfect cancellation will result in a static bias in the model. In this case, the open-loop model will be [c.f. (4)]

$$
A_{m}\left(q^{-1}\right) m(t)=q^{-k} B_{m}\left(q^{-1}\right) a(t)+d(t)+m^{b}\left(m^{*}, a^{*}\right)
$$

where $m^{b}$ is a bias term which is dependent on the operating point $\left(m^{*}, a^{*}\right)$. The 1-step-ahead moment prediction will still be given by (5), but the parameter and regression vector definitions change to

$$
\begin{aligned}
\theta= & \left(\alpha_{1} \cdots \alpha_{n a} ; b_{0} \cdots b_{n b} ; m^{b}\right)^{T} \\
\phi(t-1)= & (-m(t-1) \cdots-m(t-n a) \\
& a(t-k) \cdots a(t-k-n b) ; 1)^{T} .
\end{aligned}
$$

The optimization criterion (8) and solution (9) remain unchanged, and an estimate of the bias term $m^{b}$ is delivered as part of the solution.

A second method for dealing with the bias term resulting from imperfect cancellation, and one which avoids estimation of an additional parameter, is to remove mean levels from the data. Denoting the operating point as $\left(m^{*}, a^{*}\right)$ we have

$$
\begin{gathered}
m(t)=m^{*}+\delta m(t) \\
a(t)=a^{*}+\delta a(t)
\end{gathered}
$$

where $\delta m$ and $\delta a$ are the small deviations from the operating point. With these definitions, the average value of bias $m^{b}$ can be seen from (13) to be

$$
m^{b}=A_{m}(1) m^{*}-B_{m}(1) a^{*} \text {. }
$$

Substituting from (16)-(18) into (13), the linear transfer function around $\left(m^{*}, a^{*}\right)$ is

$$
\delta m(t)=\frac{q^{-k} B_{m}\left(q^{-1}\right)}{A_{m}\left(q^{-1}\right)} \delta a(t)+\frac{1}{A_{m}\left(q^{-1}\right)} d(t) .
$$

For parameter estimation the regression vector, therefore, becomes

$$
\begin{aligned}
\phi(t-1)= & (-\delta m(t-1) \cdots-\delta m(t-n a) \\
& \delta a(t-k) \cdots \delta a(t-k-n b))^{T}
\end{aligned}
$$

i.e., the mean levels (operating point values) are removed from the data before estimation: $\delta m(t)=m(t)-m^{*}, \delta a(t)=$ $a(t)-a^{*}$.

\section{HAMMERSTEIN MODEL}

\section{A. Recruitment Nonlinearity}

For identification of the recruitment nonlinearity we use Durfee and Maclean's impulse response method on the muscle twitch responses [12]. As they point out, this test causes little fatigue. In our "Test B," 50 pulses are applied at 1.2-s intervals; they are of ten different pulse widths and in random order. The five responses for each pulse width are averaged and the results are plotted as a surface with moment versus pulse width and time since the last pulse. The peak value of each

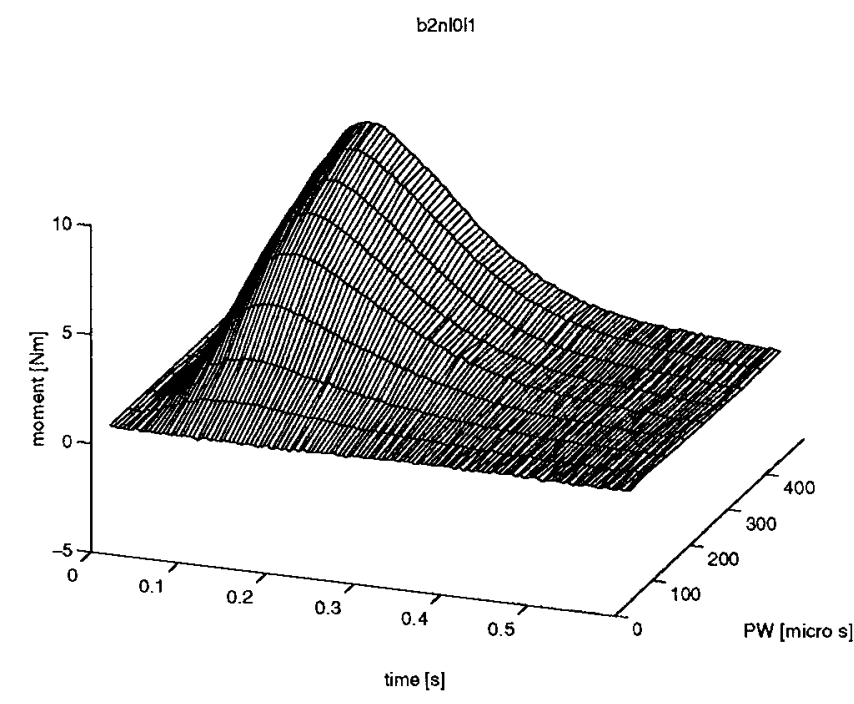

Fig. 3. Twitch response data for estimation of recruitment nonlinearity.

averaged twitch, plotted versus the stimulus pulse width, is the recruitment curve for that channel. Having found the inverse recruitment curve, we then describe the level of stimulation by its "activation level," which is the input to the inverse recruitment block ( $a(t)$ in Fig. 2).

Test B, as described above, involves the application of isolated pulses with ten distinct pulse widths of [50, 100, . 500] $\mu$ s. A typical set of averaged responses is shown in Fig. 3, which shows measured ankle moment against time, for the range of pulse widths applied.

The recruitment nonlinearity is obtained by plotting the maximum moment for each pulse width, against the pulse width. For each pulse width this results in a notional muscle activation $\tilde{a}$, normalized in the range $0-1$ Act. It is assumed that a pulse width of $500 \mu$ s corresponds to maximum activation, i.e., to $1000 \mathrm{mAct}$. Thus, to find the activation $\tilde{a}^{p}$ (in mAct) corresponding to pulse width $p$ we use

$$
\tilde{a}^{p}=\frac{m_{\max }^{p}}{m_{\max }^{500}} \cdot 1000
$$

where $m_{\max }^{500}$ is the maximum moment measured for $p=500$ and $m_{\max }^{p}$ is the maximum moment measured for the given pulse width $p$. An approximation to the recruitment curve is obtained by interpolating the experimental points, and the recruitment nonlinearity is denoted as

$$
\tilde{a}=f_{r}(p)
$$

For some desired muscle activation $a$ the estimated pulse width required is given by the inverse recruitment nonlinearity

$$
p=f_{r}^{-1}(a) \text {. }
$$

The recruitment curve obtained using this method for the test data from Fig. 3 is shown in Fig. 4. (Note that the point $(500,1000)$ has been omitted from this curve. For the pulse width $500 \mu \mathrm{s}$ an unusually high moment was recorded, resulting in a clear discontinuity in the estimated recruitment curve. We attribute this to an unknown measurement error during the test at this pulse width. One possibility is that 


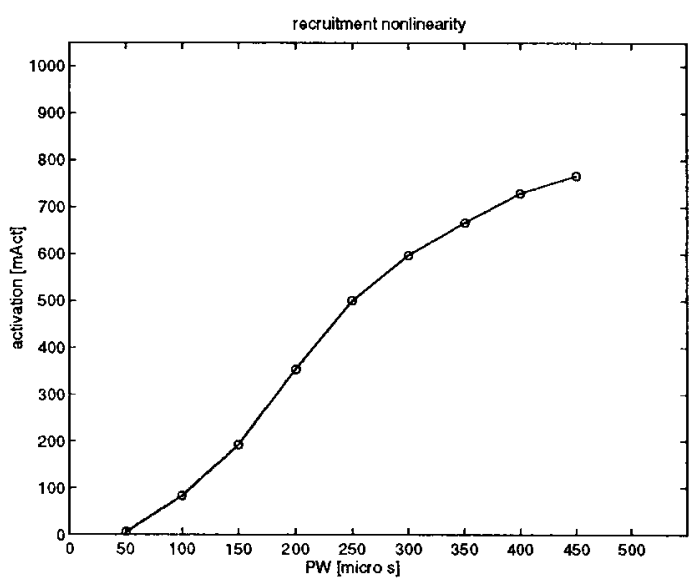

Fig. 4. Recruitment curve $f_{r}$.

the subject moved during the test. The modeling results are, however, unaffected by this.)

\section{B. Transfer-Function Estimation}

The purpose of this section is to validate the structure of the linear transfer function (4), and to find a typical set of parameters for the optimal structure. The structure of the model consists of the transfer-function order $n a$, the associated degree of the numerator polynomial, $n b$ (whereby, it is normally assumed that $n b=n a-1$ ), and the time-delay $k$. Previously, it has been found that a second-order model gives a reasonable approximation of the muscle dynamics [23], and we wished to confirm this.

The muscle was stimulated around its mid-range using a PRBS activation signal of value $500 \pm 150$ mAct. The recruitment nonlinearity was approximately cancelled as in the setup of Fig. 2. Two similar sets of data were generated: one set for model estimation, and a second set for model validation. The estimation and validation data sets are shown in Fig. 5. The test input sequence used to generate the estimation and validation data sets was kept the same, but was applied at different times. This was done in order to ensure that any differences in the estimation and validation sets were due only to unpredictable factors related to muscle physiology, and not to any input signal dependencies.

In the first validation step, the appropriate time-delay $k$ was sought: $n a$ and $n b$ were fixed as $n a=2, n b=1$, and four different models with $k=1, \cdots, 4$ were estimated according to (8)-(9) with the estimation data. For these models, the $1-, 5-$, and $\infty$-step-ahead prediction measures were calculated for the validation data, i.e., (10) with $k^{p}=1,5$, and $\infty$. (The case $k^{p}=\infty$ corresponds to pure simulation.) The validation results are summarized in Table I. It is clear that the appropriate delay is $k=1$. Note that the identified models for $k=3$ and $k=4$ were unstable, resulting in very high rms errors for pure simulation. This experimental result meets expectations since the combined delay resulting from muscle dead-time and communication protocols was anticipated to lie just below the sample time of $50 \mathrm{~ms}$, which means that the muscle responds to a change in activation within one sample period.
TABLE I

VAlidation Results: Determination of Time-Delay $k$. RMS Prediction ERrors (10) FOR THE IDENTIFIED MODELS WITH Delay $k=1,2,3$, OR 4

\begin{tabular}{l|ccc}
\hline & $\begin{array}{c}\text { avg. RMS error: } \\
\text { 1-step-ahead }\end{array}$ & $\begin{array}{c}\text { avg. RMS error: } \\
\text { 5-step-ahead }\end{array}$ & $\begin{array}{c}\text { avg. RMS error: } \\
\text { simulation }\end{array}$ \\
\hline$k=1$ & 0.521 & 2.007 & 2.374 \\
$k=2$ & 0.590 & 2.213 & 2.622 \\
$k=3$ & 0.733 & 7.137 & $\rightarrow \infty$ \\
$k=4$ & 0.734 & 6.136 & $\rightarrow \infty$ \\
\hline
\end{tabular}

The second validation step is to find the model order, na. The time delay was fixed at $k=1$ and $n a$ was varied from $1, \cdots, 4$, with $n b=n a-1$ in each case. The validation results with the four identified models for 1-, 5-, and $\infty$-stepahead predictions on the validation data are shown in Fig. 6. Each graph in Fig. 6(a)-(c) shows a clear improvement in performance as na increases from one to two. There is some improvement in each performance measure for $n a=3$, but for $n a=4$ the $\infty$-step-ahead error increases. These results suggest that the best structural parameters are $(n a, n b, k)=$ $(3,2,1)$. However, the difference in performance between models with $n a=2$ and $n a=3$ is significant only for the 1-step-ahead measure, which may indicate too strong a dependence on high-frequency information (noise) for $n a=3$. We, therefore, remain with the choice $(n a, n b, k)=(2,1,1)$ for the remainder of the paper. This brings the further advantage that the model with $n a=2$ has two parameters fewer than the model with $n a=3$.

The estimated parameters of the model with structure $(n a, n b, k)=(2,1,1)$ are

$$
\frac{q^{-k} B_{m}\left(q^{-1}\right)}{A_{m}\left(q^{-1}\right)}=\frac{q^{-1}\left(0.0040+0.0030 q^{-1}\right)}{1-1.3144 q^{-1}+0.4126 q^{-2}} .
$$

Simulation of the model against the measured muscle moment from the validation data is shown in Fig. 7, and the pole-zero configuration in Fig. 8 (the points marked "1").

It should be noted that for parameter estimation, and in the simulation result of Fig. 7, the mean levels in the input and output signals have been removed. As described in Section III-D, this avoids the need to estimate a steady-state offset term.

\section{Transfer-Function Estimation from Twitch Responses}

The PRBS-type activation signal is designed to give the best excitation of the muscle, leads to an information-rich output measurement, and is the preferred type of activation for transfer-function estimation. However, the twitch responses can in principle also be used for parametric transfer-function identification; in situations where it is critical to avoid muscle fatigue, use of the twitch response data would avoid the need for the PRBS-test. For this reason we have compared transfer functions estimated from twitch responses with the PRBSbased models. The procedure, (8) and (9), was applied to the average twitch response for a pulse width of $250 \mu \mathrm{s}$. The model thus obtained is

$$
\frac{q^{-k} B_{m}\left(q^{-1}\right)}{A_{m}\left(q^{-1}\right)}=\frac{q^{-1}\left(0.00442+0.00438 q^{-1}\right)}{1-1.1832 q^{-1}+0.3922 q^{-2}} .
$$



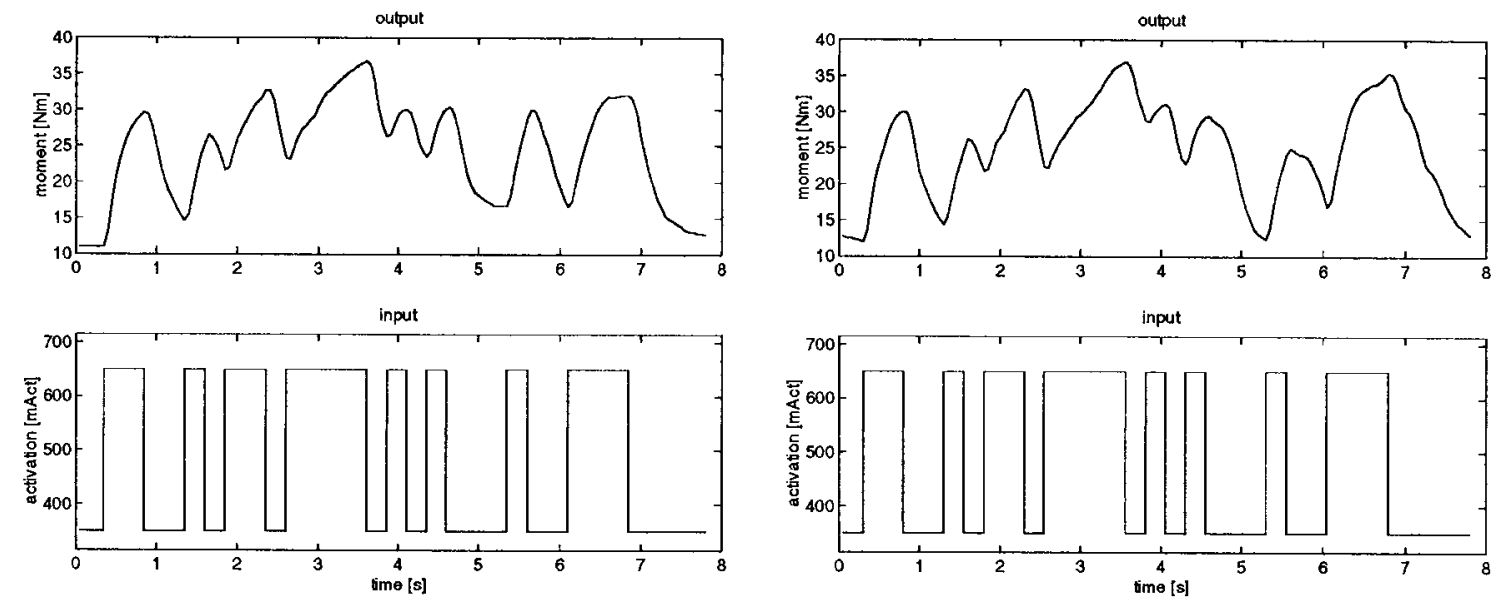

(a)

(b)

Fig. 5. PRBS identification data. (a) Estimation data set and (b) validation data set.

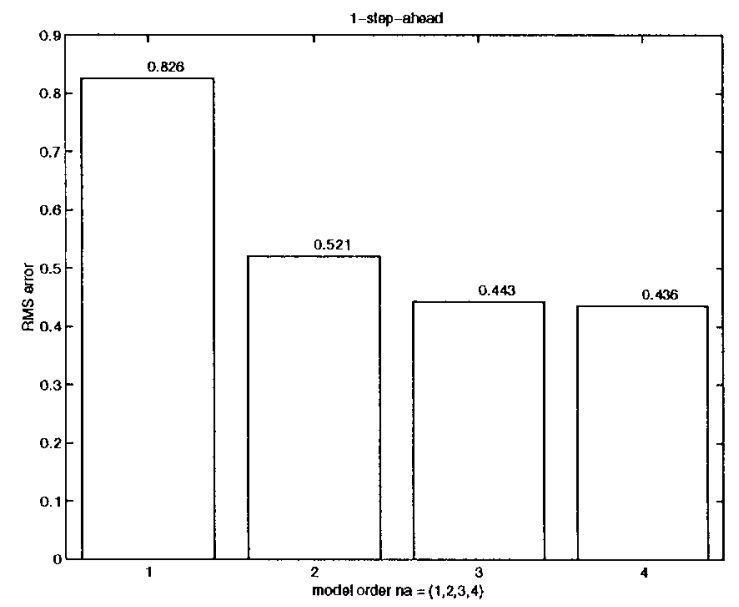

(a)

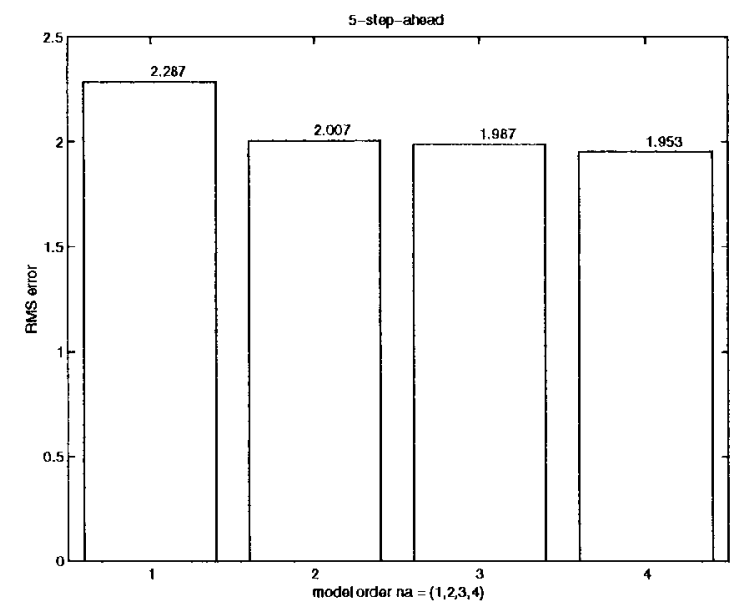

(b)

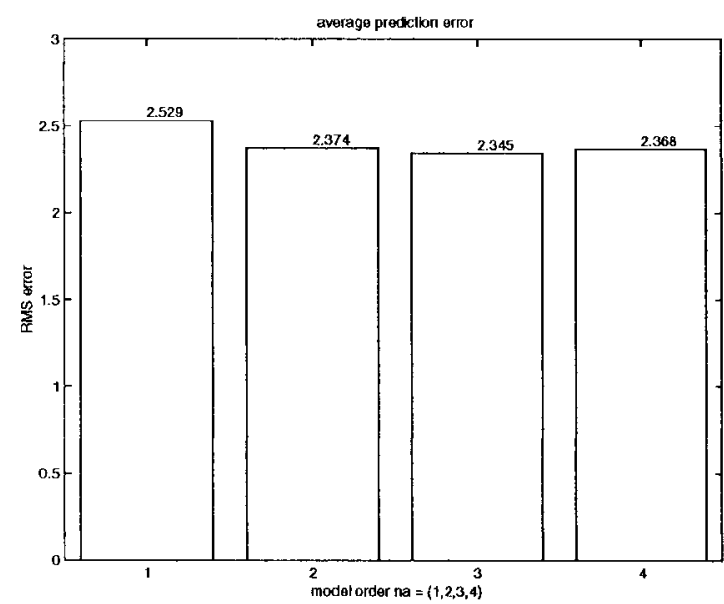

(c)

Fig. 6. Validation results: determination of model order $n a$. Each bar chart shows the rms prediction errors (10) for the identified models with $n a=1,2,3$, or 4 . (a) One-step-ahead prediction, (b) five-step-ahead prediction, and (c) pure stimulation ( $\infty$-step-ahead prediction).

The 1-, 5-, and $\infty$-step-ahead prediction errors for this model on the validation data were calculated and these are compared with those for model (24) in Table II. Quite clearly, the model identified from the twitch responses is much inferior to the PRBS-based model on this set of validation data. This is due primarily to the low dynamic information content of the twitch responses. The pole-zero configurations for models (24) and (25) are compared in Fig. 8. The number 1 in the figure denotes the PRBS-based model (24), and the number 2 denotes the twitch-response-based model (25). The 


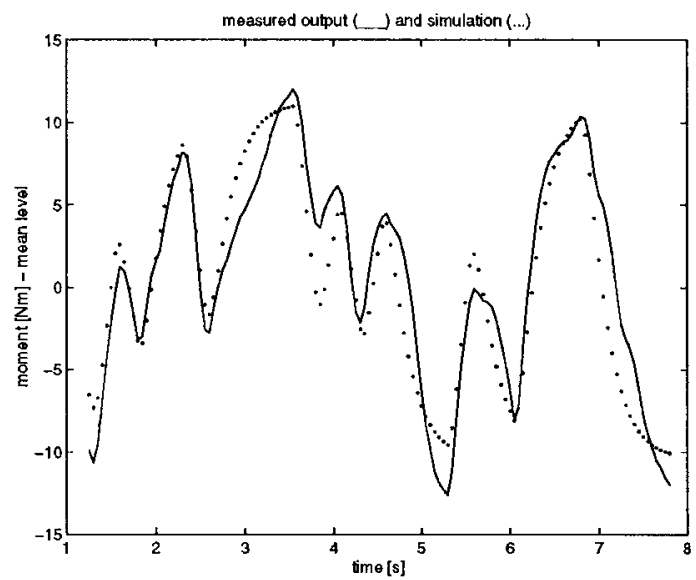

Fig. 7. Model identified from PRBS-data, $(n a, n b, k)=(2,1,1)$. Measured and simulated output. Solid line: measurement, dotted line: model.

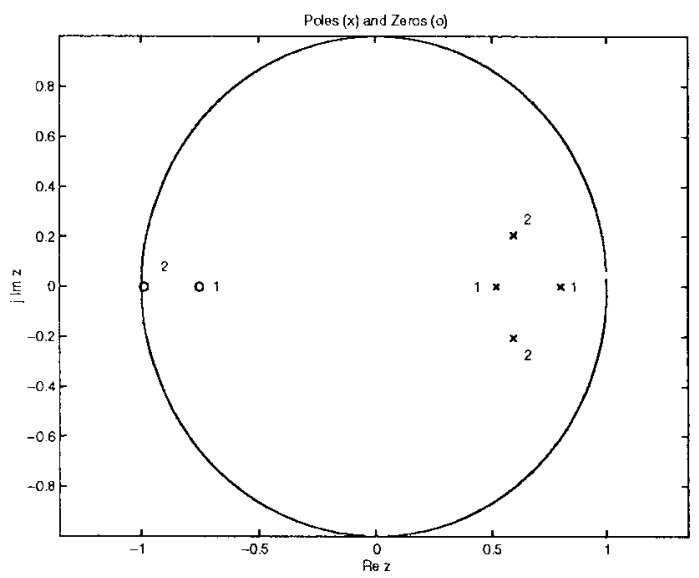

Fig. 8. Pole-zero configuration for PRBS-based model, 1, and twitch- response-based model, 2.

TABLE II

RMS PREDICTION ERRORS FOR PRBS MODEL

(24) And Twitch Response Model (25)

\begin{tabular}{l|ccc}
\hline & $\begin{array}{c}\text { RMS error: } \\
\text { 1-step-ahead }\end{array}$ & $\begin{array}{c}\text { RMS error: } \\
\text { 5-step-ahead }\end{array}$ & $\begin{array}{c}\text { RMS error: } \\
\text { simulation }\end{array}$ \\
\hline PRBS-based model (24) & 0.521 & 2.007 & 2.374 \\
twitch-based model (25) & 0.966 & 3.759 & 3.991 \\
\hline
\end{tabular}

qualitative difference is that the twitch-based model has a pair of complex-conjugate poles, thus, giving a slightly oscillatory step response. The PRBS-model has an overdamped response with two real poles. This qualitative difference is discussed further in Section V.

\section{MODEL VARIABILITY VIA LOCAL LINEARISATIONS}

In order to empirically investigate the nonlinear properties of the physical muscle, four distinct levels of PRBS-type activation signal were applied and the four corresponding local linearizations were estimated. The four activation levels referred to were as follows:

1) $125 \pm 120 \mathrm{mAct}$;

2) $375 \pm 120 \mathrm{mAct}$;

3) $625 \pm 120 \mathrm{mAct}$;

4) $875 \pm 120 \mathrm{mAct}$.

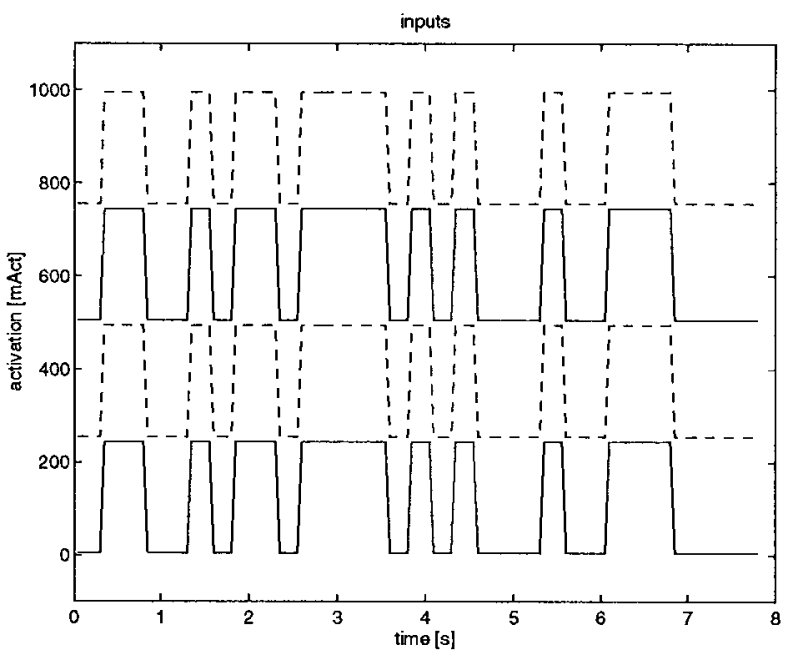

Fig. 9. PRBS activation signals, local excitation.

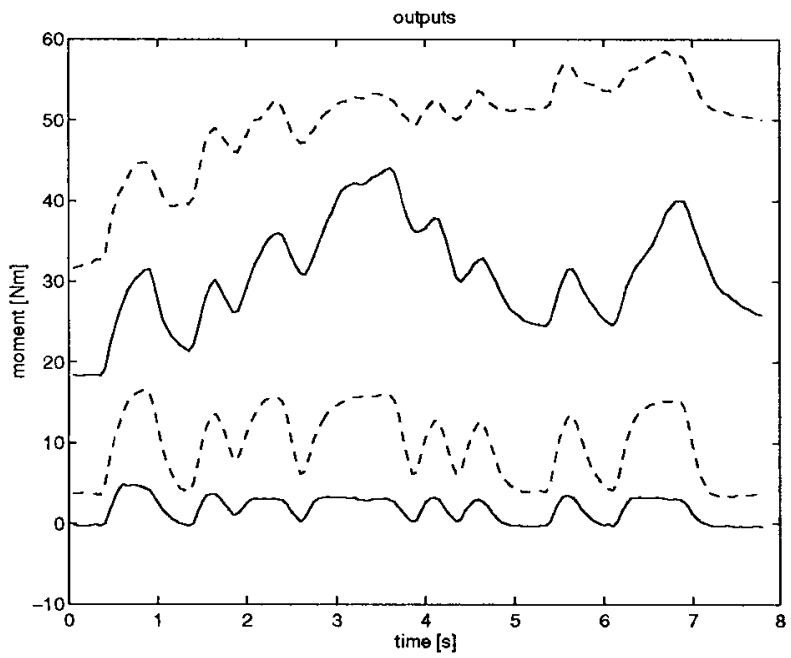

Fig. 10. Measured moments corresponding to the four local stimulation levels of Fig. 9. These data used for model estimation.

The activation signals and the corresponding measured muscle moments are shown in Figs. 9 and 10, respectively.

Since the aim here is to locally linearize the muscle, estimation of the recruitment nonlinearity, and its subsequent inversion, was not required. In fact, a linear characteristic was implemented instead of $f_{r}^{-1}$, as shown in Fig. 11, so that all nonlinear effects can be deduced by examining the local linearizations. ${ }^{2}$ The transfer functions for the four estimated local models are given in Table III, together with the steady-state gains, ${ }^{3}$ where gain $=B_{m}^{l}(1) / A_{m}^{l}(1)$ for $l=1, \cdots, 4$. Notice that the given transfer functions and gains are for the small-signal activation-moment relationship, i.e., $\delta m(t) / \delta a(t)$, where $\delta$ denotes the deviation from a fixed operating point. To obtain the pulse width-moment relationship, the given models are multiplied by the factor two

\footnotetext{
${ }^{2}$ The scaling was chosen such that an activation level of $1000 \mathrm{mAct}$ corresponds to a pulse width of $500 \mu \mathrm{s}$

${ }^{3}$ Mean levels in the input-output signals were subtracted before estimation to avoid the need to estimate operating-point-dependent offset terms (see Section III-D).
} 


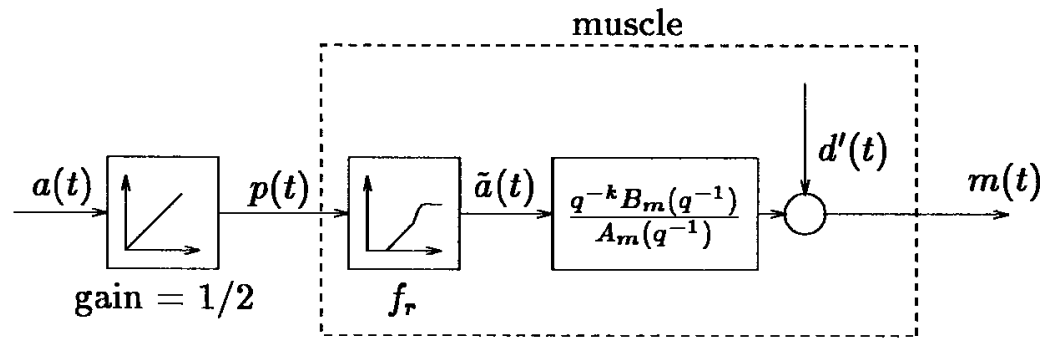

Fig. 11. Structure for identification of local linearizations, $d^{\prime}=d / A_{m}\left(q^{-1}\right)$ : no cancellation of the recruitment nonlinearity.

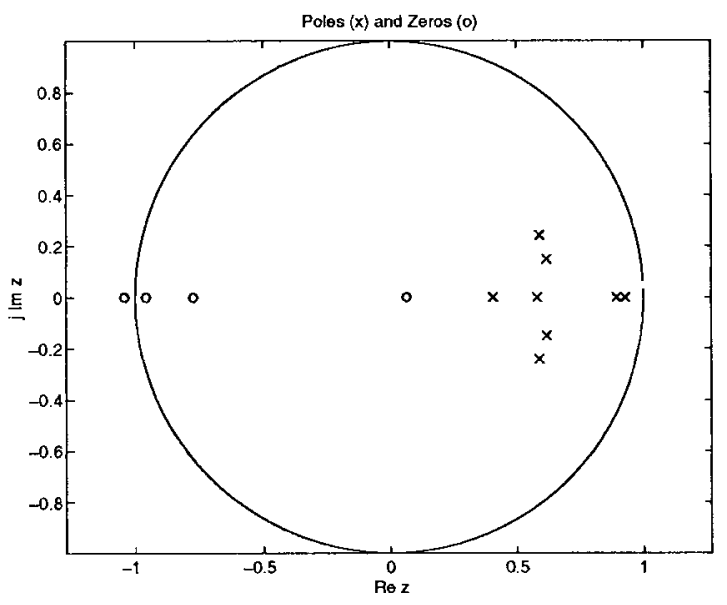

(a)

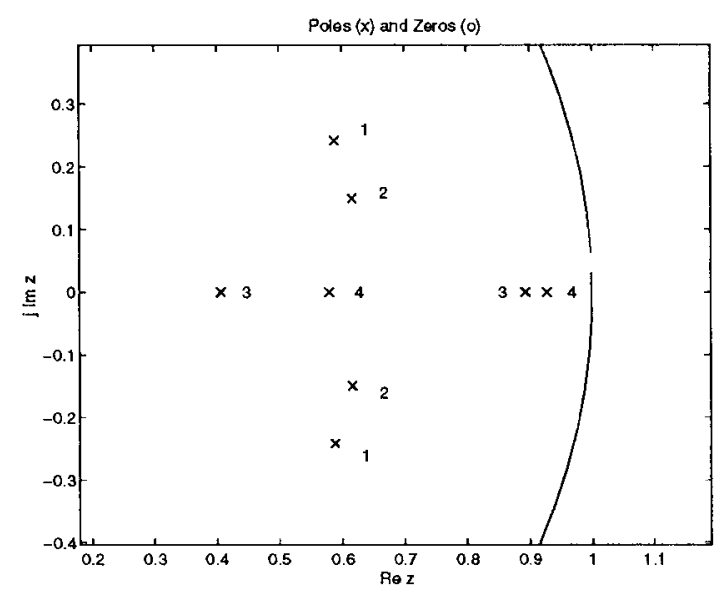

(b)

Fig. 12. (a) Pole-zero configurations for activation levels 1, 2, 3, and 4. (b) Close-up of pole positions.

TABLE III

Local Linear Models for Four Activation Levels

\begin{tabular}{ccc}
\hline Model Nr. & Transfer-function & gain \\
\hline 1 & $\frac{q^{-1}\left(0.0017+0.0017 q^{-1}\right)}{1-1.1763 q^{-1}+0.4042 q^{-2}}$ & 0.015 \\
2 & $\frac{q^{-1}\left(0.0043+0.0041 q^{-1}\right)}{1-1.2320 q^{-1}+0.4017 q^{-2}}$ & 0.050 \\
3 & $\frac{q^{-1}\left(0.0036+0.0028 q^{-1}\right)}{1-1.3010 q^{-1}+0.3639 q^{-2}}$ & 0.100 \\
4 & $\frac{q^{-1}\left(0.0025-0.0002 q^{-1}\right)}{1-1.5080 q^{-1}+0.5381 q^{-2}}$ & 0.078 \\
\hline
\end{tabular}

(i.e., the inverse of the slope of the linear characteristic of Fig. 11, where an activation of $1000 \mathrm{mAct}$ corresponds to a pulse width of $500 \mu \mathrm{s}$ ).

In Table III, the model numbers refer to the PRBS levels described above.

A consistent pattern of variability can be clearly seen from analysis of the local models. The following conclusions can be drawn regarding the qualitative properties of the muscle, as the activation level changes:

1) The pole-zero configurations for the local models are shown in Fig. 12(a), and a close-up of the pole positions, labeled according to model, in Fig. 12(b).

- At low activation, the muscle has complex-conjugate poles leading to an underdamped oscillatory response.
- As activation is increased, the poles move onto the real axis, giving an overdamped response.

- With increasing activation, the "slowest" pole (i.e., the one with the largest magnitude) migrates toward the unit circle, resulting in a slower response.

2) The variation in steady-state gain across the models is plotted in Fig. 13(a).

- The steady-state gain pattern qualitatively reflects the static Hammerstein characteristic, peaking at intermediate activation levels, and low at high and low activations (this point is discussed in depth in the following section).

3) The variation in speed of response can be seen clearly from the normalized step responses of the local transfer functions, as plotted in Fig. 13(b).

- As the activation level increases, the speed of response of the muscle decreases (as reflected in the pole migration toward the unit circle).

Thus, while the Hammerstein model to some extent reflects the variability in static gain, there are very significant changes in the dynamic properties of the muscle which are left unaccounted for in the Hammerstein structure. In fact, the speed of response of the muscle, as quantified by the rise-time in the step response of the local models, is seen to change by a factor of about five. 


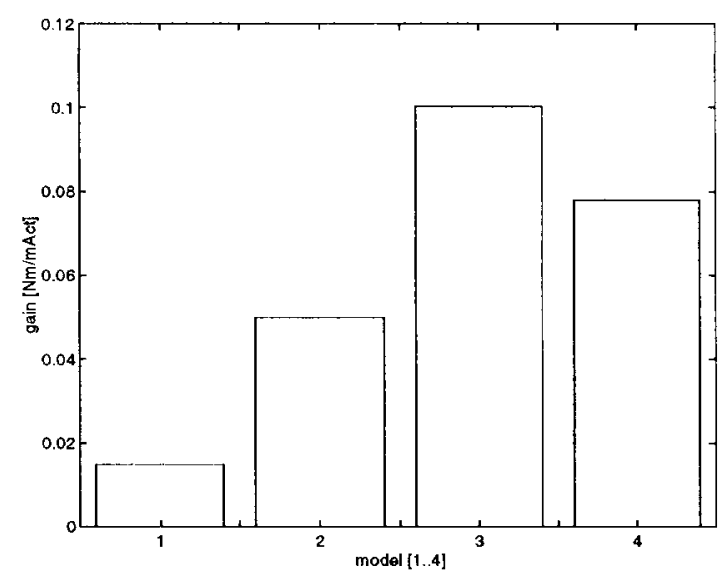

(a)

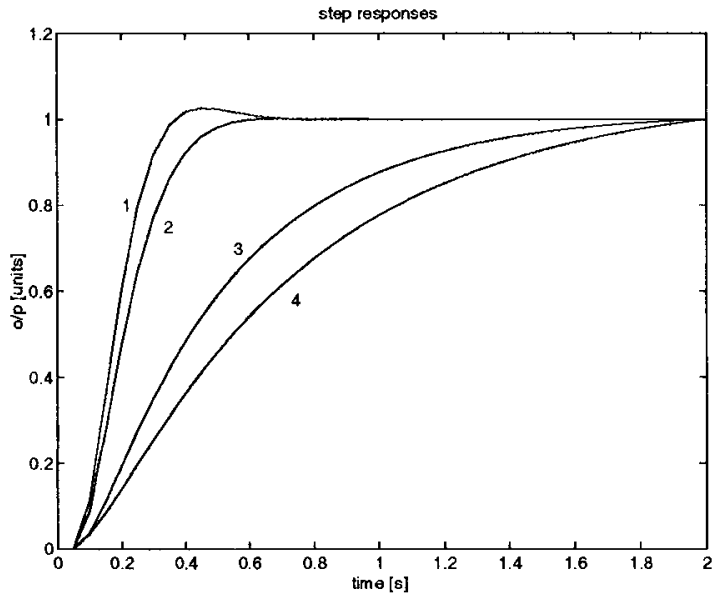

(b)

Fig. 13. Gain and time-constant properties of the four local models. (a) Steady-state gains and (b) normalized step responses

\section{COMPARISON OF HAMMERSTEIN \\ MODEL AND LOCAL LINEARISATIONS}

In Section V, we analyzed the variation in dynamic and static characteristics of the local models corresponding to different levels of muscle activation. We also noted the qualitative similarity in static gain variation between the Hammerstein model and the set of local models. In this section, we first perform some analysis which enables us to make a quantitative comparison of the gain profile, and we carry out an evaluation of the ability of the Hammerstein model to represent the muscle gain characteristics over the activation range. Second, the dynamic fidelity of the Hammerstein model over a wide operational range is compared with the combined local linearizations using a new set of validation data.

\section{A. Static Gain Properties}

Ignoring the disturbance term, the Hammerstein model of Fig. 1 is

$$
m(t)=\frac{q^{-k} B_{m}\left(q^{-1}\right)}{A_{m}\left(q^{-1}\right)} f_{r}(p(t))
$$

The small-signal behavior can be determined approximately from a first-order Taylor expansion of this expression at some fixed operating point $m(t)=m^{*}, p(t)=p^{*}$

$$
\delta m(t)=\left.\frac{q^{-k} B_{m}\left(q^{-1}\right)}{A_{m}\left(q^{-1}\right)} \cdot \frac{d f_{r}(p)}{d p}\right|_{p^{*}} \delta p(t)
$$

where the deviation variables $\delta m(t)$ and $\delta p(t)$ are defined by $\delta m(t)=m(t)-m^{*}$ and $\delta p(t)=p(t)-p^{*}$. The steady-state gain of the Hammerstein representation, denoted as $g^{h}$, is, therefore, given by

$$
g^{h}=\left.\frac{B_{m}(1)}{A_{m}(1)} \cdot \frac{d f_{r}(p)}{d p}\right|_{p^{*}} .
$$

The models in Table III, obtained by local stimulation of the muscle, each give a small-signal representation of muscle behavior

$$
\delta m(t)=\frac{q^{-k} B_{m}^{l}\left(q^{-1}\right)}{A_{m}^{l}\left(q^{-1}\right)} \delta a(t)
$$

where the deviations from activation operating point $a^{*}$ are given by $\delta a(t)=a(t)-a^{*}$. The index $l=1, \cdots, 4$ represents the four operating points used in the experiment. As noted above, for these experiments we had $p(t)=a(t) / 2$, so that the small-signal relationship between pulse width and moment is

$$
\delta m(t)=2 \frac{q^{-k} B_{m}^{l}\left(q^{-1}\right)}{A_{m}^{l}\left(q^{-1}\right)} \delta p(t) .
$$

The steady-state gain of the muscle, which for the local models we denote as $g^{l}$ is, thus, determined from

$$
g^{l}=2 \frac{B_{m}^{l}(1)}{A_{m}^{l}(1)} .
$$

The gain profile resulting from the Hammerstein model and from the set of local models can now be quantitatively compared using expressions (28) and (31); to evaluate the gain according to the Hammerstein expression (28) we take the gain of the model (24), which is $B_{m}(1) / A_{m}(1)=0.071$, and we evaluate the slope $d f_{r} / d p$ at the midpoints of the nine parts of the piecewise linear approximation to the recruitment nonlinearity depicted in Fig. 4. The gain profile thus obtained is shown as the dashed line in Fig. 14. ${ }^{4}$

Using Table III and (31), the steady-state gains of the four local models can be calculated, and these experimental points are also shown in Fig. 14 (solid line) ${ }^{5}$

Recall that the linear part of the Hammerstein model, (24), was obtained using an activation level centred at $500 \mathrm{mAct}$. From Fig. 4, this corresponds to a pulse width of $250 \mu \mathrm{s}$. It can be seen from the results in Fig. 14 that the muscle gain predicted by the two kinds of model are in close agreement

\footnotetext{
${ }^{4}$ If, instead, we used the linear transfer function (25) from the twitch responses, this graph would simply be scaled by the factor $0.04 / 0.071=$ 0.563 , where 0.04 is the steady-state gain of (25).

${ }^{5}$ Here, the four pulse widths are obtained from the four applied activation levels $(125,375,625,875)$ divided by two, i.e., $(62.5,187.5,312.5,437.5)$.
} 
TABLE IV

Average rms Prediction Errors (33) for Combined Local Models and Hammerstein Model

\begin{tabular}{c|ccc}
\hline & $\begin{array}{c}\text { avg. RMS error: } \\
\text { 1-step-ahead }\end{array}$ & $\begin{array}{c}\text { avg. RMS error: } \\
\text { 5-step-ahead }\end{array}$ & $\begin{array}{c}\text { avg. RMS error: } \\
\text { simulation }\end{array}$ \\
\hline combined local models, table 7 & 0.392 & 1.04 & 1.55 \\
Hammerstein model (24) & 0.567 & 2.15 & 2.62 \\
\hline
\end{tabular}

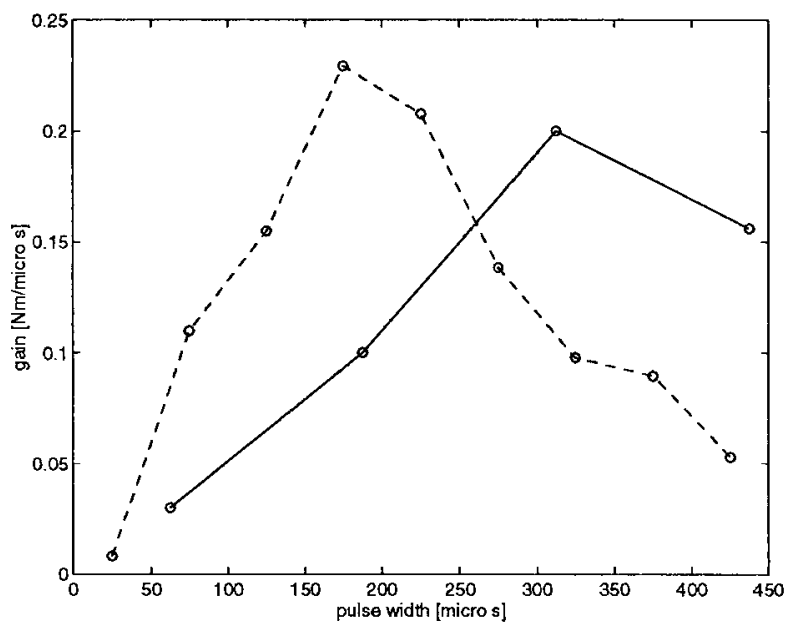

Fig. 14. Gain profiles of Hammerstein (dashed line) and local models (solid line).

only in this mid-range stimulation region. For low and high stimulation levels there is significant discrepancy in the gain profiles.

In the interpretation of this result we assume that the gain profile of the local models is accurate, due to the truly local stimulation levels used for identification of the local models. The good correspondence of the gains of the Hammerstein and local models near the centre of PRBS stimulation is as one would expect. However, the gain of the Hammerstein model is sensitive to the PRBS-stimulation region used to obtain the linear transfer function; it will be accurate only in the stimulation range where most energy is concentrated (in this case around $250 \mu \mathrm{s}$ ). Thus, we conclude that while the shape of the Hammerstein gain profile is approximately qualitatively correct, there is a strong quantitative discrepancy when viewed over the whole range of muscle stimulation.

\section{B. Comparison of Dynamic Performance}

The dynamic fidelity of the Hammerstein model over a wide operational range can be compared with the combined local linearizations using a new set of validation data. As before, four activation levels were applied to the muscle, and the resulting moments were recorded. The four activation input signals were the same as those depicted in Fig. 9, and the recorded moments this time are shown in Fig. 15.

Two kinds of model were tested on the validation data. First, for each level of activation, $l=1, \cdots, 4$, local model $l$ from Table III was applied. Second, for each level of activation, the linear transfer-function part of the Hammerstein model, (24), was applied (with cancellation of the recruitment nonlinearity).

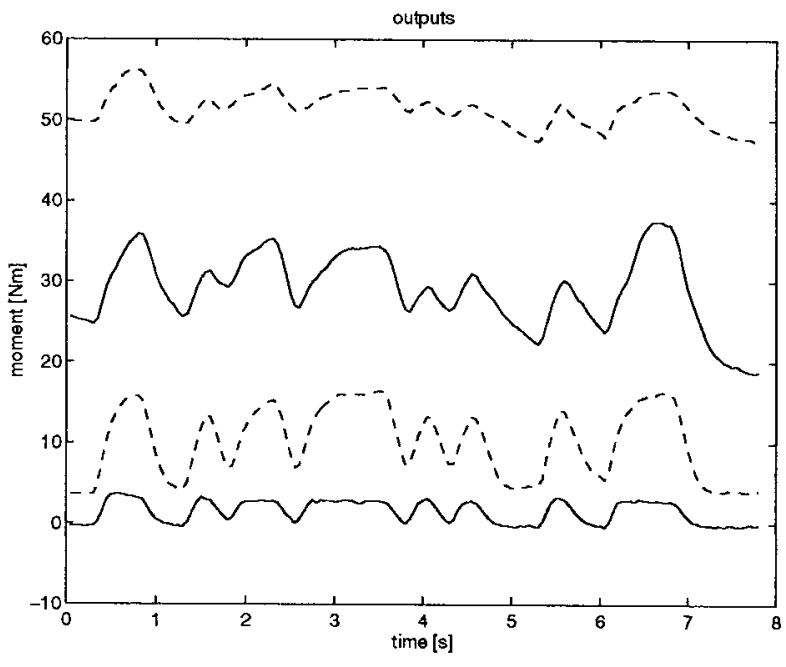

Fig. 15. Measured moments corresponding to the four local stimulation levels of Fig. 9. These data used only for validation test.

For each $l$, and each model, the $k^{p}$-step-ahead prediction error was calculated, for various $k^{p}$

$$
J_{N}^{l}\left(k^{p}, \theta\right)=\left(\frac{1}{N} \sum_{t=1}^{N}\left(m(t)-\hat{m}^{l}\left(t \mid t-k^{p}\right)\right)^{2}\right)^{\frac{1}{2}} .
$$

Here, for the combined local models, prediction $\hat{m}^{l}\left(t \mid t-k^{p}\right)$ is the prediction on data set $l$ of model $l$ from Table III. For the Hammerstein model, the prediction uses the transfer function (24) on each data set $l$. In each case, i.e., for the combined local models and the Hammerstein model, the average prediction error over all data sets was then calculated using

$$
\bar{J}\left(k^{p}, \theta\right)=\frac{1}{4} \sum_{l=1}^{4} J_{N}^{l}\left(k^{p}, \theta\right) .
$$

The results of this comparison for 1-, 5-, and $\infty$-step-ahead predictions are given in Table IV.

The results show clearly that the combined local models significantly outperform the Hammerstein model in reproducing the muscle dynamics.

\section{CONCLUSION}

We first followed the path taken by earlier investigators in identifying a Hammerstein model for the stimulated isometric muscle. Like Bawa and Stein [23], we found that a secondorder transfer function gave a good fit to stimulated calf muscles in man if there is also a transport delay, which they found to be $15-20 \mathrm{~ms}$ and we found to be less than $50 \mathrm{~ms}$ 
(one sample period). We found that a good model structure is $(n a, n b, k)=(2,1,1)$. Durfee and Maclean [12] pointed out that if avoiding fatigue is important, the frequency response of the muscle can be identified from the twitch responses, though Allin and Inbar [11] preferred to excite the muscle with PRBS activation, which we find gives a better model. The tests we used are quick. Test B takes 1 min and each PRBS test, at one mean level, takes $10 \mathrm{~s}$.

The use of the Hammerstein model to represent electrically stimulated muscle is based on the assumption that the dynamics are independent of activation level. As explained in Section I, one would expect that for normal muscles, stimulated with rectangular pulses of current, the slower motor units would be recruited at high activation and, therefore, that the dynamics will change with activation level. This is shown in Figs. 12 and 13, the speed of response falling dramatically by a factor of five. The gain of the linear part of the Hammerstein model is found using the PRBS response to stimulus pulses around one average width. Consequently, the small-signal gain of the model is quantitatively correct for activation by pulses close to this width, but it is in error by factors of up to three at other pulse widths (Fig. 14). Finally, it was shown that the dynamic performance of the combined local models is significantly better than that of a Hammerstein model.

These large discrepancies of the Hammerstein model imply that one of two possible choices must be made for stimulation controllers. If a linear controller followed by an inverse recruitment curve is to be used [4], the design must be robust enough to accomodate the actual range of gain and speed of the muscles. Local models can be used to design such a robust controller but this will only be achieved by loss of performance. On the other hand, high performance may be achieved by adopting a nonlinear control strategy [24], [25].

Local models can be joined into a single model by interpolation [26], [27] and this naturally leads to corresponding controllers in which the controller gain and dynamics are progressively altered by scheduling [28]. Our results show that activation is an appropriate scheduling variable for such a controller, which we expect could be designed and implemented quite easily (see [24] for a simulation study). Perhaps the chief outstanding question is whether this black-box approach is the best replacement for the Hammerstein muscle models and their controllers, or whether some more physiological model will be better [29]. This should depend on the time taken to perform the identification tests, the range of operating conditions which the model describes and the desired accuracy, and whether the calculations can be performed in real time on practical stimulators. These questions will form the basis of our future research.

\section{REFERENCES}

[1] E. Henneman and C. Olson, "Relations between structure and function in the design of skeletal muscle," J. Neurophysiol., vol. 28, pp. 581-598, 1965.

[2] J. T. Mortimer, "Motor prostheses," in Handbook of Physiology-The Nervous System II, J. M. Brookhart, V. B. Mountcastle, V. B. Brooks, and S. R. Geiger, Eds. Bethesda, MD: Amer. Physiological Soc., 1981, pp. $155-187$.
[3] W. Durfee, "Model identification in neural prostheses system," in Neural Prostheses, R. B. Stein, P. H. Peckham, and D. B. Popovic, Eds. New York: Oxford Univ. Press, 1992, pp. 58-87.

[4] P. Marmarelis and V. Marmarelis, Analysis of Physiological Systems. New York: Plenum, 1978.

[5] A. Hammerstein, "Nichlineare Integralgleichungen nebst Anwendungen," Acta Mathematica, vol. 54, pp. 117-176, 1930.

[6] I. Hunter and M. Korenburg, "The identification of nonlinear biological systems: Wiener and Hammerstein cascade models," Biol., Cyber., vol. 55, pp. 135-144, 1986.

[7] L. Vodovnik, W. Crochetiere, and J. Reswick, "Control of skeletal joints by electrical stimulation," Med., Biol. Eng., vol. 5, pp. 97-109, 1967.

[8] A. Trnkoczy, T. Bajd, and M. Malezic, "A dynamic model of the ankle joint under functional electrical stimulation in free movement and isometric conditions," J. Biomech., vol. 9, pp. 509-519, 1978.

[9] G. Wilhere, P. Crago, and H. Chizeck, "Design and evaluation of a digital closed-loop controller for the regulation of muscle force by recruitment modulation," Trans. IEEE Biomed. Eng., vol. BME-32, pp. 668-676, Sept. 1985

[10] L. Bernotas, P. Crago, and H. Chizeck, "A discrete-time model of electrically stimulated muscle," Trans. IEEE Biomed. Eng., vol. BME33, pp. 829-838, 1986.

[11] J. Allin and G. Inbar, "FNS parameter selection and upper limb characterization," Trans. IEEE Biomed. Eng., vol. BME-33, pp. 809-817, 1986.

[12] W. K. Durfee and K. E. Maclean, "Methods of estimating the isometric recruitment curve of electrically stimulated muscle," Trans. IEEE Biomed. Eng., vol. 36, pp. 654-667, 1989.

[13] R. Stein and M. Oguztoreli, "A model of whole muscles incorporating functionally important non linearities," in Non Linear Phenomena in Mathematical Sciences. New York: Academic, 1982.

[14] N. Donaldson, F. M. D. Barr, G. F. Phillips, and T. A. Perkins, "Unsupported standing of paraplegics by stimulation of the plantarflexors: Some results from the wobbler apparatus," in Neuroprosthetics: From Basic Research to Clinical Application, A. Pedotti, M. Ferrarin, J. Quintern, and R. Riener, Eds. New York: Springer-Verlag, 1996.

[15] K. J. Hunt, M. Munih, N. Donaldson, and F. M. D. Barr, "Optimal control of ankle joint moment: Toward unsupported standing in paraplegia," Trans. IEEE Automat. Contr., vol. 43, pp. 819-832, June 1998.

[16] K. J. Hunt, M. Munih, and N. Donaldson, "Feedback control of unsupported standing in paraplegia. Part I: Optimal control approach," Trans. IEEE Rehab. Eng., vol. 5, pp. 331-340, Dec. 1997.

[17] M. Munih, N. Donaldson, K. J. Hunt, and F. M. D. Barr, "Feedback control of unsupported standing in paraplegia. Part II: Experimental results," Trans. IEEE Rehab. Eng., vol. 5, pp. 341-352, Dec. 1997.

[18] G. F. Phillips, J. R. Adler, and S. J. G. Taylor, "A portable programmable eight-channel surface stimulator," in Proc. Ljubljana FES Conf., 1993, pp. $166-168$

[19] L. Ljung and T. Söderström, Theory and Practice of Recursive Identification. London, UK: MIT Press, 1983.

[20] L. Ljung, System Identification-Theory for the User. Englewood Cliffs, NJ: Prentice-Hall, 1987.

[21] M. Munih, K. J. Hunt, and N. Donaldson, "LQG control for the ankle joint moment," presented at ICMMB Conf., Ljubljana, Slovenia, 1996.

[22] M. Munih, K. J. Hunt, N. Donaldson, and F. M. D. Barr, "LQG moment control in the paraplegic's ankle joint," presented at 18th Int. Conf. IEEE Eng., in Medicine and Biology Society, Amsterdam, the Netherlands, 1996.

[23] P. Bawa and R. Stein, "Frequency response of human soleus muscle," J. Neurophysiol., vol. 39, pp. 788-793, 1976.

[24] H. Gollee and K. J. Hunt, "Nonlinear modeling and control of electrically stimulated muscle: A local model network approach," Int. J. Contr., vol. 68, no. 6, pp. 1259-1288, 1997.

[25] H. Gollee, K. J. Hunt, N. Donaldson, and J. C. Jarvis, "Modeling of electrically stimulated muscle," in Multiple Model Approaches to Modeling and Control, R. Murray-Smith and T. A. Johansen, Eds.. London, U.K.: Taylor and Francis, 1997, ch. 3, pp. 101-120.

[26] T. A. Johansen and B. A. Foss, "A NARMAX model representation for adaptive control based on local model," Modeling, Identification, Contr., vol. 13 , no. 1 , pp. $25-39,1992$.

[27] _ "Constructing NARMAX models using ARMAX models," Int. J. Contr., vol. 58, no. 5, pp. 1125-1153, 1993.

[28] K. J. Hunt and T. A. Johansen, "Design and analysis of gain-scheduled control using local controller networks," Int. J. Contr., vol. 66, no. 5 , pp. 619-651, 1997.

[29] R. Riener and J. Quintern, "Physiologically based model of muscle activation evaluated by electrical stimulation," in Proc. 9th Int. Conf. Mech. in Med., and Biol., 1996, pp. 230-223. 


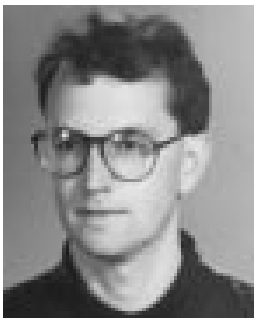

Kenneth J. Hunt (S'87-M'87) received the 1st class honors B.Sc. degree in electrical and electronic engineering in 1984 and the Ph.D. degree in control theory in 1987, both from the University of Strathclyde, Glasgow, Scotland.

From 1989-1992 he held a Royal Society of Edinburgh Personal Research Fellowship, and during that period was with the Department of Mechanical Engineering at the University of Glasgow. He moved to Daimler-Benz in November 1992. From 1987-1989 he was a Research Scientist with BBN Systems and Technologies (the European division of Bolt, Beranek, and Newman Inc.). From 1992-1997 he was a Research Scientist and Project Leader with Daimler-Benz Systems Technology Research Berlin, part of Daimler-Benz corporate research. He is Professor of Mechanical Engineering in the Centre for Systems and Control at the University of Glasgow, Scotland. $\mathrm{He}$ is also a Visiting Professor at the Department of Automatic Control and Systems Engineering at the University of Sheffield.

In August 1992, Dr. Hunt was Local Arrangements Chair at the IEEE International Symposium on Intelligent Control in Glasgow. A Chartered Engineer, he is a member of the Institution of Electrical Engineers and contributes to the IFAC Technical Committee on Adaptive Systems. He is chairman of IFAC's recently formed Technical Committee on Fuzzy and Neural Systems, and is Associate Editor of the IEEE TRANSACtions on CONTROL SyStems TEChNOLOGY.

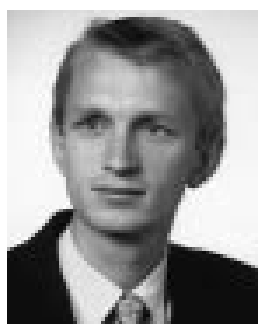

Marko Munih (M'88) received the B.Sc. (1986) and M.Sc. (1989) degrees in electrical engineering from the University of Ljubljana, Slovenia. He received the D.Sc. degree in electrical engineering in 1993 for work on controlling muscle activity with respect to the femur and tibia bone loading.

In 1989 he was appointed as Teaching Assistant at the Faculty of Electrical Engineering. His research interests are focused in functional electrical stimulation of paraplegic lower extremities with surface electrode systems, including measurement, control, biomechanics, and electrical circuits. From 1995 to 1996 he was Research Assistant with the Implanted Devices Group in the Department of Medical Physics and Bioengineering at University College London, where he did research on unsupported standing of paraplegics and development of the sensory amplifier. Currently, he is a faculty member at the Faculty of Electrical Engineering, University of Ljubljana, Slovenia.

$\mathrm{He}$ is member of the IFMBE, IFESS and IFAC and serves as an international referee for several journals in the biomedical engineering field.

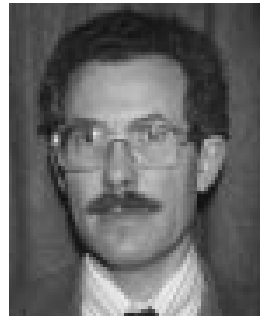

Nick de N. Donaldson studied engineering at Cambridge University, Cambridge, U.K. After a time spent designing integrated circuits at the General Electric Company, London, U.K., he joined the Medical Research Council Neurological Prostheses Unit, London, U.K., in 1976. Since then he has worked on the use of surgically implanted devices for restoring paralyzed limb function. His Ph.D. degree dissertation was received from London University.

Since 1992, he has been Head of the Implanted Devices Group at University College London, London, U.K., which has a close collaboration with the Spinal Injuries Units at the Royal National Orthopaedic Hospital and Salisbury Hospital in England. His research interests include implant technology, implanted orthopaedic instrumentation, the biomechanics of standing, biomechanical instrumentation, nerve and nerve root stimulation, feedback control in FES, and use of ENG signals in control.

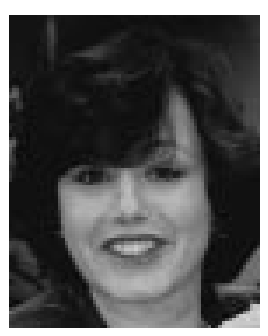

Fiona M. D. Barr studied for the diploma in physiotherapy at the Bristol School of Physiotherapy, Bristol, U.K., and for the M.Sc. degree at Kings College London, London, England.

She is a Research Physiotherapist at the Royal National Orthopaedic Hospital, Stanmore, England. She has had much experience of the application of FES to patients with spinal cord injury. Her particular interest is in the effect of electrical stimulation on spasticity. She is currently preparing the Ph.D. thesis on this quantification of spasticity. 